\title{
Decreased expression of granulocyte-macrophage colony-stimulating factor is associated with adverse clinical outcome in patients with gastric cancer undergoing gastrectomy
}

\author{
HAO LIU ${ }^{1 *}$, CHAO LIN $^{1 *}$, ZHENBIN SHEN $^{1 *}$, HENG ZHANG $^{1}$, \\ HONGYONG HE ${ }^{1}, \mathrm{HE} \mathrm{LI}^{1}$, JING QIN ${ }^{1}$, XINYU QIN ${ }^{1}$, JIEJIE XU ${ }^{2 *}$ and YIHONG SUN ${ }^{1 *}$ \\ ${ }^{1}$ Department of General Surgery, Zhongshan Hospital; ${ }^{2}$ Department of Biochemistry and Molecular Biology, \\ School of Basic Medical Sciences, Fudan University, Shanghai 200032, P.R. China
}

Received December 22, 2015; Accepted May 26, 2017

DOI: $10.3892 / 01.2017 .6738$

\begin{abstract}
Previous studies have revealed the clinical significance of tumor-associated macrophages (TAMs) in gastric cancer, whereas the role of the cytokines that orchestrate TAM polarization in gastric cancer remains elusive. The present study aimed to evaluate the prognostic value of granulocyte-macrophage colony-stimulating factor (GM-CSF) expression in patients with gastric cancer. Intratumoral GM-CSF expression was investigated by immunohistochemical staining in 408 retrospectively enrolled patients. Kaplan-Meier analysis and Cox regression models were used to evaluate the prognostic value of GM-CSF expression. Predictive nomograms were generated to predict the overall survival and disease-free survival rates of the patients. Decreased intratumoral GM-CSF expression was identified, and indicated a poorer clinical outcome for patients with gastric cancer, particularly in advanced stages. Intratumoral GM-CSF expression may provide an additional risk stratification for the prognosis of patients with gastric cancer based on the Tumor-Node-Metastasis (TNM) staging system. Cox multivariate analysis identified GM-CSF expression as an independent prognostic factor for overall survival and disease-free survival time. The generated nomograms performed well in predicting the 3-and 5-year clinical outcome
\end{abstract}

Correspondence to: Dr Yihong Sun, Department of General Surgery, Zhongshan Hospital, Fudan University, 180 Fenglin Road, Shanghai 200032, P.R. China

E-mail: sun.yihong@zs-hospital.sh.cn

Dr Jiejie Xu, Department of Biochemistry and Molecular Biology, School of Basic Medical Sciences, Fudan University, 138 Yixueyuan Road, Shanghai 200032, P.R. China

E-mail: jjxufdu@fudan.edu.cn

*Contributed equally

Key words: gastric cancer, granulocyte-macrophage colony-stimulating factor, overall survival, disease-free survival, prognostic biomarker, nomogram of patients with gastric cancer. In conclusion, GM-CSF is a potential independent prognostic indicator for patients with gastric cancer, which may be integrated with TNM staging systems to improve the predictive accuracy for clinical outcome, particularly in advanced tumors.

\section{Introduction}

Despite decreased incidence and mortality rates in previous decades, gastric cancer remains the fourth most common malignancy and the third leading cause of cancer-associated mortality worldwide $(1,2)$. Due to the mild and atypical symptoms at the early stage, $>80 \%$ of the patients are clinically diagnosed at an advanced stage, which generally indicates a poor outcome $(1,3)$. For the stratification of patient risk, the underlying molecular and cellular processes during gastric carcinogenesis are ignored in the widely-used Union for International Cancer Control/American Joint Committee on Cancer (UICC/AJCC) Tumor Node Metastasis (TNM) staging systems (4), while previous evidence has demonstrated its heterogeneity, with an unpredictable clinical outcome (4). There is an urgent requirement to illuminate the molecular events involved in the development and progression of gastric cancer, making it possible to improve disease prognosis and provide novel therapeutic targets for treatment.

Since the 19th century, cancer has been associated with inflammation. Emerging evidence has revealed that inflammation serves an important role in the initiation, development and progression of human malignancy $(5,6)$. As the most abundant immune cell in the tumor microenvironment, macrophages have received attention for their pro-tumoral role by facilitating neoangiogenesis in the primary tumor site, and promoting metastasis in distant sites (7-9). Macrophages that infiltrated into the tumor microenvironment were primed to adopt a pro-tumoral M2-phenotype rather than a tumoricidal M1-phenotype (10). In the process of the polarization and activation of macrophages, a variety of chemokines were identified, of which granulocyte-macrophage colony-stimulating factor (GM-CSF) may be the essential orchestrator $(10,11)$. The role of GM-CSF, also termed CSF-2, in the tumor microenvironment is controversial. Certain studies revealed that GM-CSF 
promoted tumorigenesis via stimulating the epithelial release of VEGF (12), while others stated that GM-CSF released by tumor cells was associated with improved survival (13) in colorectal cancer. In breast cancer, GM-CSF was identified to inhibit cancer growth and metastasis (14), and GM-CSF triggered and maintained the alternative activation of tumor-associated macrophages (TAM), and promoted tumor growth and angiogenesis in glioma (15). However, the clinical significance of intratumoral GM-CSF and its prognostic value in gastric cancer remains obscure.

The prognostic role of diametrically polarized TAMs in gastric cancer has been identified in our previous study (16). The present study aimed to investigate the expression of GM-CSF in gastric cancer and its correlation with the clinicopathological characteristics and clinical outcomes, including overall survival (OS) and disease-free survival (DFS) times. In addition, nomograms were generated to evaluate the 3- and 5-year DFS and OS rates for the patients with gastric cancer following surgery.

\section{Patients and methods}

Clinical specimens. A total of 408 patients diagnosed with gastric cancer at Zhongshan Hospital, Fudan University (Shanghai, China) from January 1, 2008 to December 31, 2008 were enrolled in the present study. The male:female ratio was 2.37 and the median age of the patients was 60 (range, 27-88) years old. Written informed consent from each patient was obtained, and the use of human specimens was approved by the Clinical Research Ethics Committee of Zhongshan Hospital. All the patients received a radical resection (R0) with a D2 lymphadenectomy from the same surgical team and the resultant formalin-fixed paraffin-embedded surgically resected specimens were used in the present study. The specimens were fixed in $10 \%$ formalin for $12 \mathrm{~h}$ at room temperature and were embedded in paraffin for $4 \mathrm{~h}$ at $60^{\circ} \mathrm{C}$. The section width was $5 \mu \mathrm{m}$. No patients had received any anti-cancer therapy prior to surgery. The clinicopathological and baseline demographic characteristics of the patients were retrospectively collected, including age, sex, tumor size, tumor histological classification (17), Lauren's classification (18) and TNM stage (4). A total of 2 independent gastroenterology pathologists from the Department of Pathology of Zhongshan Hospital provided reassessments for the tumor stage according to the 7th Edition of the UICC/AJCC TNM staging system (4). DFS was defined as the time from the date of surgery to the date of recurrence or the last visit. OS was defined as the time from the date of surgery to the date of death from all causes or the last visit.

Tissue microarray and immunohistochemistry. The construction of the tissue microarray and the immunohistochemical protocols were as previously described (19). An anti-GM-CSF antibody (dilution, 1:100 at $5 \mu \mathrm{g} / \mathrm{ml}$; cat. no. ab9741, Abcam, Cambridge, MA, USA) was used as the primary antibody in the immunohistochemical analysis. The semi-quantitative $\mathrm{H}$-score, which ranged from 0 to 300 , was calculated by multiplying the staining intensities ( 0 , negative; 1 , weak; 2 , moderate; 3 , strong) by the distribution areas (percentage of positive staining cancer cells, $0-100 \%$ ) at each intensity level for each sample. A total of 2 independent observers who were blinded to the patient outcomes and clinicopathological characteristics provided the evaluation of the immunostaining.

Statistical analysis. The cut-off point for the definition of high/low expression subgroups was determined by X-tile plot analysis (20). SPSS 19.0 (IBM Corp., Armonk, NY, USA) was used to perform the analyses. The Pearson $\chi^{2}$ test or Kruskal-Wallis test was used to compare categorical variables. Continuous variables were analyzed with an unpaired Student's t-test. Survival estimates were conducted with Kaplan-Meier curves, and statistical significance was determined using the log-rank test. Multivariable Cox proportional hazards models were used to identify the independent prognosticator. A nomogram was generated by R software v3.2.2 with 'rms' package ( $R$ Foundation for Statistical Computing, Vienna, Austria). Calibration plots for 3- and 5-year survival rates were constructed to examine the performance characteristics of the generated nomograms. The prognostic accuracy was measured by calculating the Harrell's concordance indices (c-indices). All statistical analyses were two-sided, and $\mathrm{P}<0.05$ was considered to indicate a statistically significant difference.

\section{Results}

Associations between GM-CSF immunohistochemical expression and the clinicopathological features. Immunohistochemical staining analysis was performed in 408 clinical specimens resected from primary tumor sites. GM-CSF staining greatly varied in intensity in the tumor tissues (Fig. 1A and B). The positive staining of GM-CSF was observed primarily in the cytoplasm and/or on the membrane of neoplastic epithelia, and partially in the stroma. According to the semi-quantitative $\mathrm{H}$-score, 239 (58.6\%) cases were included in the GM-CSF low expression group. The clinicopathological features of the patients dichotomized by intratumoral GM-CSF expression are summarized in Table I. No significant association was identified between GM-CSF expression patterns and the clinicopathological features.

Prognostic evaluation of GM-CSF expression in patients with gastric cancer. The Kaplan-Meier method and log-rank tests were performed to assess the association between GM-CSF expression and clinical outcome in patients with gastric cancer. At the last follow-up, the mean duration of OS was 40.2 months (median, 44.5 months) and DFS was 37.9 months (median, 41.0 months). Patients with low GM-CSF expression were more likely to exhibit poorer survival [Hazard ratio (HR), 2.26; 95\% confidence interval (CI), 1.65-3.11; $\mathrm{P}<0.001$; Fig. 1C] and suffer from earlier recurrence (HR, 1.68; 95\% CI, 1.26-2.26; $\mathrm{P}=0.001$; Fig. 1D) compared with those with high expression. The median DFS and OS times for the GM-CSF low expression subgroup were 52 and 55 months, respectively, while those for the high expression subgroup were 30.1 and 34 months, respectively.

In order to eliminate the effects of tumor stage on prognosis, Kaplan-Meier analysis was also applied to compare OS according to GM-CSF expression in different TNM stages. A statistically significant difference was identified in advanced stages of tumors when stratified by GM-CSF expression levels (Fig. 2A and B), while the DFS and OS of the patients with 
Table I. Correlations between GM-CSF expression and clinicopathological features in patients with gastric cancer ( $\mathrm{n}=408$ ).

\begin{tabular}{|c|c|c|c|c|}
\hline \multirow[b]{2}{*}{ Characteristics } & \multirow[b]{2}{*}{ All patients } & \multicolumn{3}{|c|}{ GM-CSF expression } \\
\hline & & Low & High & P-value ${ }^{a}$ \\
\hline Age, years & & & & 0.358 \\
\hline Mean \pm SD & $60.0 \pm 11.7$ & $60.5 \pm 11.8$ & $59.4 \pm 11.5$ & \\
\hline Sex & & & & 0.178 \\
\hline Male & 287 & 162 & 125 & \\
\hline Female & 121 & 77 & 44 & \\
\hline Tumor size, cm & & & & 0.073 \\
\hline Mean \pm SD & $3.81 \pm 2.16$ & $3.98 \pm 2.23$ & $3.59 \pm 2.05$ & \\
\hline Differentiation & & & & 0.811 \\
\hline Well differentiated & 17 & 9 & 8 & \\
\hline Moderately differentiated & 150 & 88 & 62 & \\
\hline Poorly differentiated ${ }^{\mathrm{b}}$ & 241 & 142 & 99 & \\
\hline Lauren's classification & & & & 0.998 \\
\hline Intestinal & 261 & 153 & 108 & \\
\hline Diffuse & 96 & 56 & 40 & \\
\hline Mixed & 51 & 30 & 21 & \\
\hline Depth of invasion & & & & 0.081 \\
\hline $\mathrm{T} 1$ & 70 & 34 & 36 & \\
\hline $\mathrm{T} 2$ & 57 & 34 & 23 & \\
\hline $\mathrm{T} 3$ & 75 & 43 & 32 & \\
\hline $\mathrm{T} 4$ & 206 & 128 & 78 & \\
\hline Lymph node metastasis & & & & 0.280 \\
\hline No & 153 & 84 & 69 & \\
\hline $\mathrm{N} 1$ & 45 & 26 & 19 & \\
\hline $\mathrm{N} 2$ & 78 & 49 & 29 & \\
\hline N3 & 132 & 80 & 52 & \\
\hline pTNM stage & & & & 0.113 \\
\hline $\mathrm{I}$ & 97 & 51 & 46 & \\
\hline II & 93 & 53 & 40 & \\
\hline III & 218 & 135 & 83 & \\
\hline Adjuvant chemocherapy ${ }^{\mathrm{c}}$ & & & & 0.916 \\
\hline Yes & 245 & 143 & 102 & \\
\hline No & 163 & 96 & 67 & \\
\hline
\end{tabular}

${ }^{\mathrm{a}} \chi^{2}$ test, Kruskal-Wallis test or Student's t-test was performed. $\mathrm{P}<0.05$ was considered to indicate a statistically significant difference. ${ }^{\mathrm{b}}$ Signet-cell carcinoma and mucinous adenocarcinoma included. 'Patients with adjuvant chemotherapy received at least one cycle of 5-fluorouracil-based chemotherapy. pTNM, pathological Tumor-Node-Metastasis; SD, standard deviation; GM-CSF, granulocyte-macrophage colony-stimulating factor.

TNM I stage tumors were not significantly different (Fig. 2C and D).

In the univariate Cox regression analysis of OS, intratumoral GM-CSF expression was defined as a prognostic factor $(\mathrm{P}<0.001)$. Multivariable Cox proportional hazards models that included depth of tumor invasion, lymph node metastasis, GM-CSF expression and Lauren's classification as co-variables were constructed. For OS, depth of tumor invasion $(\mathrm{P}<0.001)$, lymph node metastasis $(\mathrm{P}<0.001)$, adjuvant chemotherapy $(\mathrm{P}<0.001)$ and $\mathrm{GM}-\mathrm{CSF}$ expression $(\mathrm{P}<0.001)$ were identified to be independent prognostic factors for patients with gastric cancer, while for DFS, depth of tumor invasion $(\mathrm{P}<0.001)$, lymph node metastasis $(\mathrm{P}<0.001)$, Lauren's classification $(\mathrm{P}=0.029)$, adjuvant chemotherapy $(\mathrm{P}=0.001)$ and $\mathrm{GM}-\mathrm{CSF}$ expression $(\mathrm{P}=0.009)$ were identified to be independent prognostic factors (Table II).

Predictive nomogram for OS in gastric cancer patients. In order to provide a quantitative assessment for outcomes of patients with gastric cancer, 2 nomograms were constructed to provide a more sensitive prognostic model (Figs. 3A and 4A). The factors incorporated in the nomogram were independent 
Table II. Multivariate analysis for OS and DFS in patients with gastric cancer $(n=408)$.

\begin{tabular}{|c|c|c|c|c|c|c|}
\hline \multirow[b]{2}{*}{ Variables } & \multicolumn{3}{|c|}{ OS } & \multicolumn{3}{|c|}{ DFS } \\
\hline & HR & $95 \% \mathrm{CI}$ & P-value ${ }^{a}$ & HR & $95 \% \mathrm{CI}$ & P-value ${ }^{a}$ \\
\hline Depth of tumor invasion $(\mathrm{T} 3+4 \mathrm{vs} . \mathrm{T} 1+2)$ & 3.674 & $2.292-5.889$ & $<0.001$ & 4.704 & 2.846-7.774 & $<0.001$ \\
\hline Lymph node metastasis ( $\mathrm{N} 2+3$ vs. $\mathrm{N} 0+1)$ & 2.827 & $1.982-4.033$ & $<0.001$ & 3.128 & 2.199-4.449 & $<0.001$ \\
\hline Lauren classification (diffused + mixed vs. intestinal) & 1.201 & $0.897-1.608$ & 0.218 & 1.376 & $1.032-1.834$ & 0.029 \\
\hline GM-CSF expression (low vs. high) & 2.109 & $1.531-2.904$ & $<0.001$ & 1.631 & $1.130-2.354$ & 0.009 \\
\hline Adjuvant chemotherapy $^{\mathrm{b}}$ (no vs. yes) & 2.297 & $1.656-3.186$ & $<0.001$ & 1.630 & $1.214-2.190$ & 0.001 \\
\hline
\end{tabular}

${ }^{\text {aD }}$ ata obtained from the Cox proportional hazards model. $\mathrm{P}<0.05$ was considered to indicate a statistically significant difference; ${ }^{\mathrm{b}} \mathrm{Patients}$ with adjuvant chemotherapy received at least one cycle of 5-fluorouracil-based chemotherapy. DFS, disease-free survival; OS, overall survival; GM-CSF, granulocyte-macrophage colony-stimulating factor; HR, hazard ratio; CI, confidence interval.
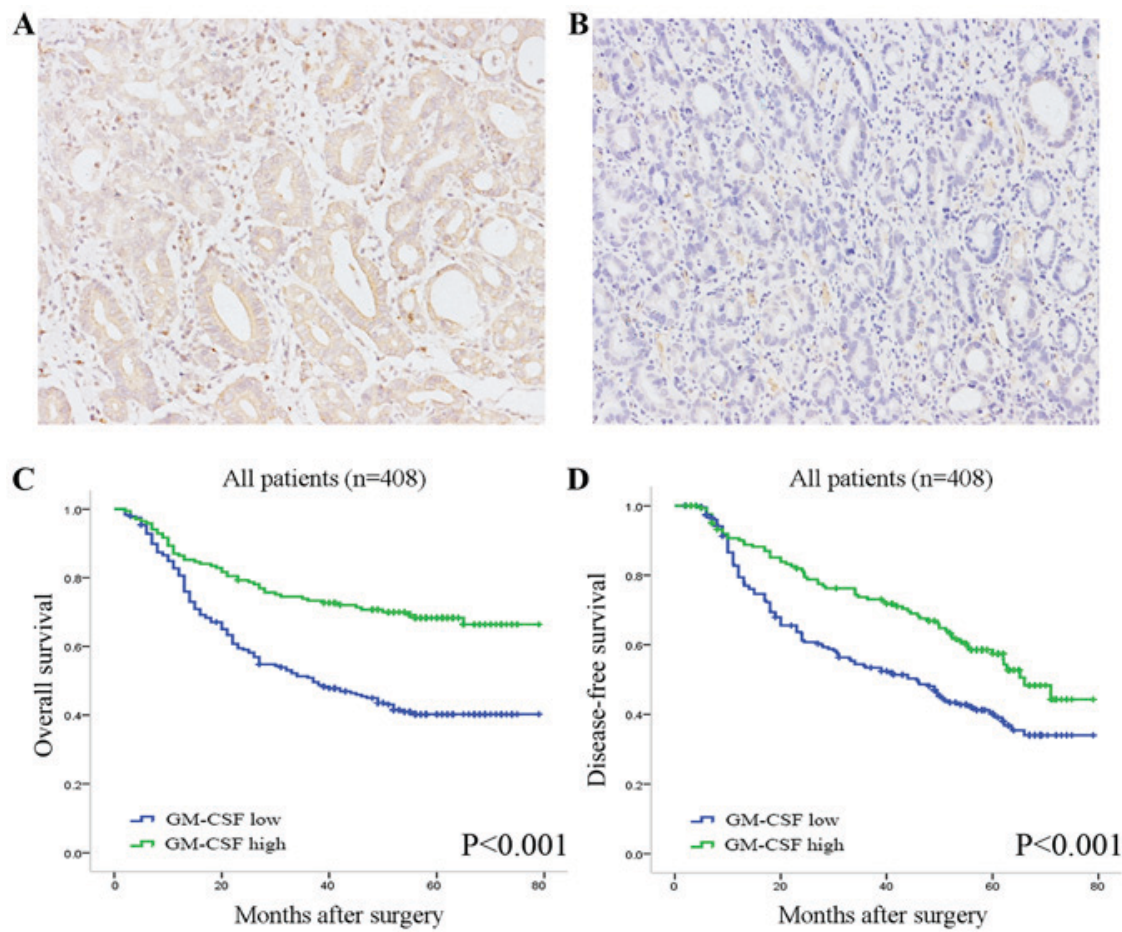

Figure 1. Patients with gastric cancer with decreased intratumoral GM-CSF expression exhibit a poorer clinical outcome. Representative images of immunostained tumor tissue with (A) high GM-CSF expression and (B) low GM-CSF expression. Magnification, x200. Kaplan-Meier analysis according to intratumoral GM-CSF expression in all patients with gastric cancer for (C) overall survival and (D) disease-free survival. GM-CSF, granulocyte-macrophage colony-stimulating factor.

factors for OS selected subsequent to multivariate analysis, with the exception of adjuvant chemotherapy, to generate a model that only included the characteristics of the tumor without artificial interventions. A higher number of total points predicted a poorer prognosis. The total point was raised by the addition of the score of each factor for each patient correspondingly. Calibration curves for the internal validation of the nomogram predictions of 3-and 5-year survival rate were constructed, and the nomograms performed well with the ideal model (Fig. 3B and C; Fig. 4B and C). Harrell's c-index for the generated nomogram was 0.714 (95\% CI, 0.679-0.749) for OS and 0.743 for DFS (95\% CI, 0.708-0.778). The area under the receiver operating curve of the generated nomogram for OS was 0.804 , which was significantly larger compared with that of TNM stage $(0.742 ; \mathrm{P}<0.001 ;$ Fig. 3D). The area under the receiver operating curve of the generated nomogram for DFS was 0.807, which was also larger compared with that of TNM stage (0.779; $\mathrm{P}=0.015 ;$ Fig. 4D). These data indicated that the nomograms performed well in predicting the OS of the patients.

\section{Discussion}

Previous studies have revealed the important role of tumor-associated macrophages in the process of tumor development and progression $(10,21)$. Our previous study also demonstrated the prognostic value of infiltrated polarized macrophages in gastric cancer (16). However, the role of cytokines that are involved in 

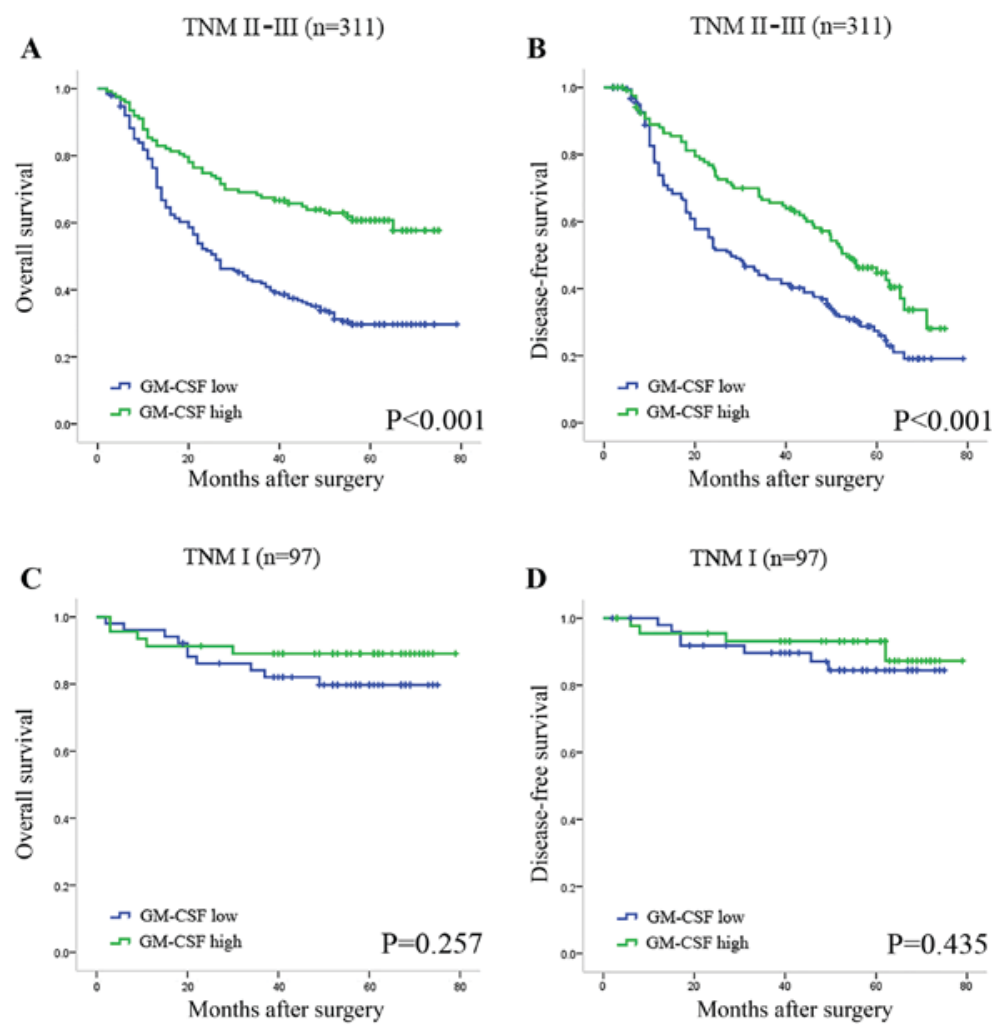

Figure 2. Intratumoral GM-CSF expression refines prognostic information to the TNM staging system. Kaplan-Meier analysis for (A) OS and (B) DFS according to intratumoral GM-CSF expression in patients with TNM II-III stage tumors. Kaplan-Meier analysis for (C) OS and (D) DFS according to intratumoral GM-CSF expression in patients with TNM I stage tumors. GM-CSF, granulocyte-macrophage colony stimulating factor; TNM, Tumor-Node-Metastasis; OS, overall survival; DFS, disease-free survival.

the orchestration of the polarization of TAM remains controversial. To the best of our knowledge, the present study is the first study that identified intratumoral GM-CSF expression as an independent prognostic factor for patients following gastrectomy. In addition, the generated nomograms performed better compared with the TNM staging system in predicting the DFS and OS for the patients.

Much attention has been paid to TAMs for their crucial role in the carcinogenesis of various tumors (10). For gastric cancer, Ohno et al (22) stated that the aggregation of TAMs within the tumor nest demonstrated a tumoricidal effect, while Ishigami et al (23) identified that the presence of TAMs in tumor tissue was correlated with an adverse prognosis. However, our previous study revealed that the infiltration of M2-polarized TAM in tumor tissue was associated with a favorable outcome, while M1-TAM infiltration exhibited the opposite effect (16). These raised lead to the investigation of the cytokines that are involved in the polarization of TAMs. In a previous study, a high expression of $\mathrm{C}-\mathrm{C}$ motif chemokine 2 (CCL-2) in the tumor tissue of gastric cancer was revealed to be associated with the poor OS of the patients (24). The present study identified that high intratumoral expression of GM-CSF was correlated with an improved clinical outcome. Therefore, it is conceivable that increased GM-CSF may promote M2 to M1 polarization of macrophages, which would impede infiltration and invasion of the primary tumor, while CCL-2 possibly directed the opposite polarization.

Since Lauren's classification was introduced in 1965 (18), debates have continued on whether this may provide risk stratification in patients with gastric cancer. Lauren diffuse-type gastric cancer is frequently associated with a mutation of the Cadherin 1 (CDH1) gene (25-27). Mutation or loss and methylation of $\mathrm{CDH} 1$ leads to the aberrant expression of E-cadherin, disturbing the normal cell-cell adhesion (27). Therefore, gastric cancer cells of diffuse-type may be more likely to disperse and disseminate. This may provide a potential explanation for the results of the present study, which suggest that Lauren's classification was an independent prognostic factor for DFS, but not for OS.

The prognostic value of GM-CSF expression in gastric cancer, particularly in advanced tumors, was investigated in the present study. According to intratumoral GM-CSF expression, patients were separated into two subgroups. GM-CSF expression was demonstrated to be an independent adverse prognosticator for OS and DFS in patients with gastric cancer. Furthermore, nomograms were constructed by integrating GM-CSF expression, depth of tumor invasion and lymph node metastasis status to provide a prediction for the 3 - and 5 -year OS and DFS rates of the patients. Calibration plots and c-indices indicated that the generated nomograms performed better than the TNM staging system in terms of discriminating between patients with different clinical outcomes. However, a limitation exists that the study design is retrospective in nature and the number of patients enrolled was relatively small. A large, multi-center, prospective study is required to validate these results.

It is known that anticancer therapies, including cytotoxic drugs, radiotherapy and targeted agents, depend on the activation 

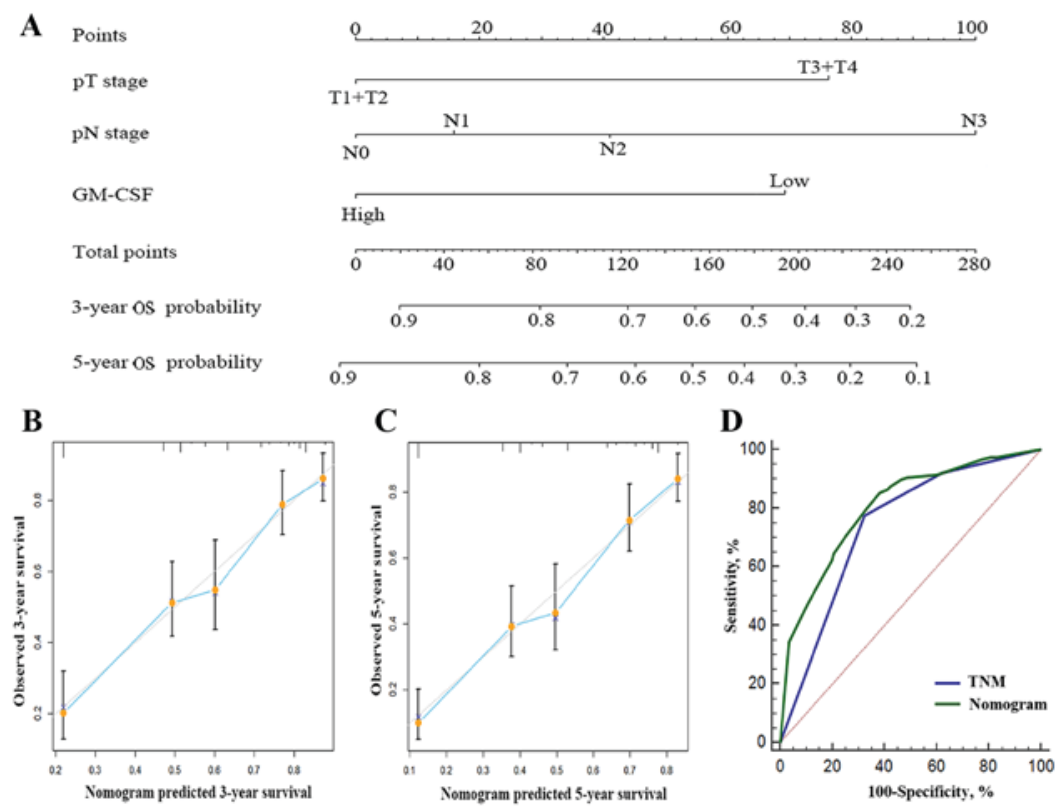

Figure 3. Nomogram generated from independent prognostic factors predicts OS. (A) Nomogram for predicting clinical outcome integrated GM-CSF expression (high/low) with depth of tumor invasion (T1+T2/T3+T4) and lymph node metastasis (N0/N1/N2/N3). (B) Calibration plot for nomogram predicting 3 -year OS rate. (C) Calibration plot for nomogram predicting 5-year OS rate. (D) Receiver operating characteristic analysis of the sensitivity and specificity for the predictive value for OS of the generated nomogram and the TNM staging system. GM-CSF, granulocyte-macrophage colony-stimulating factor; TNM, Tumor-Node-Metastasis; OS, overall survival; p, pathological.

A

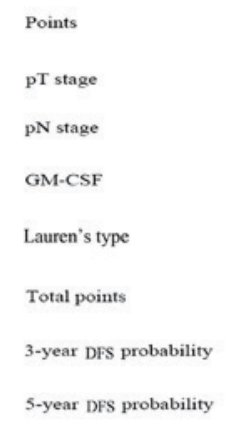

B

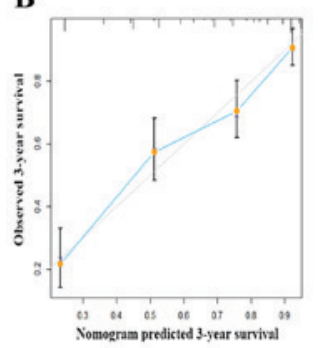

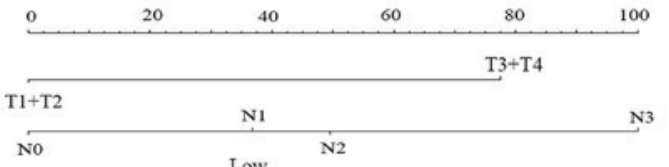
Low

High Dif. Mixed

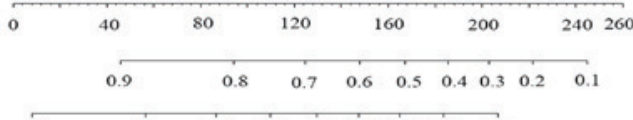

$\begin{array}{llllllllll}0.9 & 0.8 & 0.7 & 0.6 & 0.5 & 0.4 & 0.3 & 0.2 & 0.1\end{array}$

C

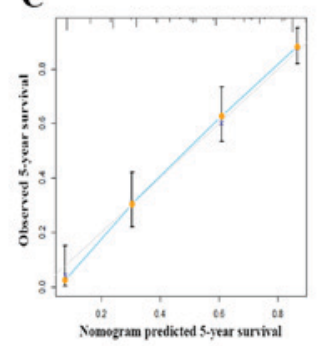

Figure 4. Nomogram generated from independent prognostic factors predicts DFS. (A) Nomogram for predicting clinical outcomes integrated GM-CSF expression (high/low) with depth of tumor invasion (T1+T2/T3+T4), lymph node metastasis (N0/N1/N2/N3) and Lauren's classification (diffuse + mixed/intestinal). (B) Calibration plot for nomogram predicted 3-year DFS rate. (C) Calibration plot for nomogram predicted 5-year DFS rate. (D) Receiver operating characteristic analysis of the sensitivity and specificity for the predictive value for DFS of the generated nomogram and the TNM staging system. GM-CSF, granulocyte-macrophage colony-stimulating factor; TNM, Tumor-Node-Metastasis; DFS, disease-free survival.

of anticancer immune responses (28). Studies on the reversion of M1/M2 polarization, the prognostic value of TAMs $(29,30)$ and GM-CSF expression, as in the present study, have raised the possibility that by altering the level of cytokines, for example, increasing the concentration of GM-CSF in the tumor microenvironment, the reversal of the polarization of TAMs may provide a novel target for the treatment of gastric cancer.
In conclusion, intratumoral expression of GM-CSF in gastric cancer has been identified as an independent prognostic factor for OS and DFS. Furthermore, intratumoral GM-CSF expression may be integrated with the depth of tumor invasion and lymph node metastasis status to provide improved risk stratification for patients with gastric cancer with different prognosis, particularly in advanced stages. 


\section{Acknowledgements}

The present study was funded by grants from the National Key Projects for Infectious Diseases of China (grant nos. 2012ZX10002012-007 and 2016ZX10002018-008), the National Natural Science Foundation of China (grant nos. 31100629, 31270863, 81372755, 31470794, 81401988, $81402082,81402085,81471621,81472227,81472376,31570803$, 81501999 and 81572352) and the Program for New Century Excellent Talents in University (grant no. NCET-13-0146).

\section{References}

1. Torre LA, Bray F, Siegel RL, Ferlay J, Lortet-Tieulent J and Jemal A: Global cancer statistics, 2012. CA Cancer J Clin 65: 87-108, 2015

2. Mayer RJ, Venook AP and Schilsky RL: Progress against GI cancer during the American society of clinical oncology's first 50 years. J Clin Oncol 32: 1521-1530, 2014.

3. Chen WQ, Zheng RS, Zhang SW, Zeng HM and Zou XN: The incidences and mortalities of major cancers in China, 2010. Chin J Cancer 33: 402-405, 2014.

4. Washington K: 7th edition of the AJCC cancer staging manual: Stomach. Ann Surg Oncol 17: 3077-3079, 2010.

5. Mantovani A, Allavena P, Sica A and Balkwill F: Cancer-related inflammation. Nature 454: 436-444, 2008.

6. Coussens LM and Werb Z: Inflammation and cancer. Nature 420 860-867, 2002.

7. Wynn TA, Chawla A and Pollard JW: Macrophage biology in development, homeostasis and disease. Nature 496: 445-455, 2013.

8. Wu H, Xu JB, He YL, Peng JJ, Zhang XH, Chen CQ, Li W and Cai SR: Tumor-associated macrophages promote angiogenesis and lymphangiogenesis of gastric cancer. J Surg Oncol 106 462-468, 2012.

9. Pollard JW: Trophic macrophages in development and disease. Nat Rev Immunol 9: 259-270, 2009.

10. Noy R and Pollard JW: Tumor-associated macrophages: From mechanisms to therapy. Immunity 41: 49-61, 2014.

11. Murray PJ, Allen JE, Biswas SK, Fisher EA, Gilroy DW, Goerdt S, Gordon S, Hamilton JA, Ivashkiv LB, Lawrence T, et al: Macrophage activation and polarization: Nomenclature and experimental guidelines. Immunity 41: 14-20, 2014.

12. Wang Y, Han G, Wang K, Liu G, Wang R, Xiao H, Li X, Hou C, Shen B, Guo R, et al: Tumor-derived GM-CSF promotes inflammatory colon carcinogenesis via stimulating epithelial release of VEGF. Cancer Res 74: 716-726, 2014.

13. Nebiker CA, Han J, Eppenberger-Castori S, Iezzi G, Hirt C, Amicarella F, Cremonesi E, Huber X, Padovan E, Angrisani $\mathrm{B}$, et al: GM-CSF production by tumor cells is associated with improved survival in colorectal cancer. Clin Cancer Res 20: 3094-3106, 2014

14. Eubank TD, Roberts RD, Khan M, Curry JM, Nuovo GJ, Kuppusamy P and Marsh CB: Granulocyte macrophage colony-stimulating factor inhibits breast cancer growth and metastasis by invoking an anti-angiogenic program in tumor-educated macrophages. Cancer Res 69: 2133-2140, 2009.

15. Sielska M, Przanowski P, Wylot B, Gabrusiewicz K, Maleszewska M, Kijewska M, Zawadzka M, Kucharska J, Vinnakota K, Kettenmann H, et al: Distinct roles of CSF family cytokines in macrophage infiltration and activation in glioma progression and injury response. J Pathol 230: 310-321, 2013.
16. Zhang H, Wang X, Shen Z, Xu J, Qin J and Sun Y: Infiltration of diametrically polarized macrophages predicts overall survival of patients with gastric cancer after surgical resection. Gastric Cancer 18: 740-50, 2015.

17. Japanese Gastric Cancer Association: Japanese classification of gastric carcinoma: 3rd English edition. Gastric Cancer 14: 101-112, 2011.

18. Lauren P: The two histological main types of gastric carcinoma: Diffuse and so-called intestinal-type carcinoma. An attempt at a histo-clinical classification. Acta Pathol Microbiol Scand 64: 31-49, 1965

19. Zhu XD, Zhang JB, Zhuang PY, Zhu HG, Zhang W, Xiong YQ, Wu WZ, Wang L, Tang ZY and Sun HC: High expression of macrophage colony-stimulating factor in peritumoral liver tissue is associated with poor survival after curative resection of hepatocellular carcinoma. J Clin Oncol 26: 2707-2716, 2008.

20. Camp RL, Dolled-Filhart M and Rimm DL: X-tile: A new bio-informatics tool for biomarker assessment and outcome-based cut-point optimization. Clin Cancer Res 10: 7252-7259, 2004.

21. Sica A, Schioppa T, Mantovani A and Allavena P: Tumour-associated macrophages are a distinct M2 polarised population promoting tumour progression: Potential targets of anti-cancer therapy. Eur J Cancer 42: 717-727, 2006.

22. Ohno S, Inagawa H, Dhar DK, Fujii T, Ueda S, Tachibana M, et al: The degree of macrophage infiltration into the cancer cell nest is a significant predictor of survival in gastric cancer patients. Anticancer Res 23, 5015-5022, 2003.

23. Ishigami S, Natsugoe S, Tokuda K, Nakajo A, Okumura $H$, Matsumoto M, et al. Tumor-associated macrophage (TAM) infiltration in gastric cancer. Anticancer Res 23, 4079-4083, 2003.

24. Liu H, Shen Z, Wang X, Zhang H, Qin J, Qin X, Xu J and Sun Y: Increased expression of $\mathrm{C}-\mathrm{C}$ motif ligand 2 associates with poor prognosis in patients with gastric cancer after gastrectomy. Tumor Biol 37: 3285-3293, 2016.

25. Oda T, Kanai Y, Oyama T, Yoshiura K, Shimoyama Y, Birchmeier W, Sugimura T and Hirohashi S: E-cadherin gene mutations in human gastric carcinoma cell lines. Proc Natl Acad Sci USA 91: 1858-1862, 1994

26. Yoshiura K, Kanai Y, Ochiai A, Shimoyama Y, Sugimura T and Hirohashi S: Silencing of the E-cadherin invasion-suppressor gene by $\mathrm{CpG}$ methylation in human carcinomas. Proc Natl Acad Sci USA 92: 7416-7419, 1995.

27. Hansford S, Kaurah P, Li-Chang H, Woo M, Senz J, Pinheiro H, Schrader KA, Schaeffer DF, Shumansky K, Zogopoulos G, et al: Hereditary diffuse gastric cancer syndrome: CDH1 mutations and beyond. JAMA Oncol 1: 23-32, 2015.

28. Galluzzi L, Senovilla L, Zitvogel L and Kroemer G: The secret ally: Immunostimulation by anticancer drugs. Nat Rev Drug Discov 11: 215-233, 2012.

29. Guiducci C, Vicari AP, Sangaletti S, Trinchieri G and Colombo MP: Redirecting in vivo elicited tumor infiltrating macrophages and dendritic cells towards tumor rejection. Cancer Res 65: 3437-3446, 2005.

30. Saccani A, Schioppa T, Porta C, Biswas SK, Nebuloni M, Vago L, Bottazzi B, Colombo MP, Mantovani A and Sica A: P50 nuclear factor-kappaB overexpression in tumor-associated macrophages inhibits M1 inflammatory responses and antitumor resistance. Cancer Res 66: 11432-11440, 2006. 